Old Dominion University

ODU Digital Commons

\title{
Inverted Geometry Ceramic Insulators in High Voltage DC Electron Guns for Accelerators
}

\author{
C. Hernandez-Garcia \\ G. Palacios-Serrano \\ P. Adderley \\ D. Bullard \\ J. Grames
}

See next page for additional authors

Follow this and additional works at: https://digitalcommons.odu.edu/physics_fac_pubs

Part of the Nuclear Engineering Commons, and the Power and Energy Commons

\section{Original Publication Citation}

Hernandez-Garcia, C., G. Palacios-Serrano, P. Adderly, D. Bullard, J. Grames, M. A. Mamun, M. Poelker, M. Stutzman, R. Suleiman, Y. Wang and S. A. K. Wijethunga. (2021). Inverted geometry ceramic insulators in high voltage DC electron guns for accelerators. Paper presented at the 2021 IEEE Conference on Electrical Insulation and Dielectric Phenomena (CEIDP). doi: 10.1109/CEIDP50766.2021.9705333

This Conference Paper is brought to you for free and open access by the Physics at ODU Digital Commons. It has been accepted for inclusion in Physics Faculty Publications by an authorized administrator of ODU Digital Commons. For more information, please contact digitalcommons@odu.edu. 


\section{Authors}

C. Hernandez-Garcia, G. Palacios-Serrano, P. Adderley, D. Bullard, J. Grames, Md. Abdullah Mamun, M. Poelker, M. Stutzman, R. Suleiman, Y. Wang, and S.A.K. Wijethunga 


\title{
Inverted Geometry Ceramic Insulators in High Voltage DC Electron Guns for Accelerators
}

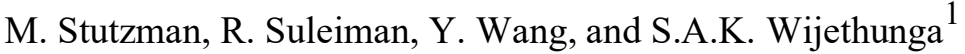

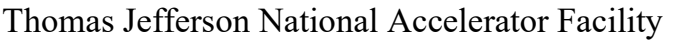

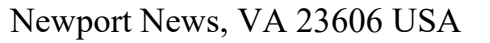

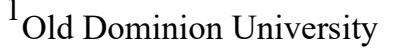

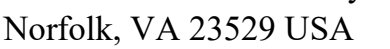

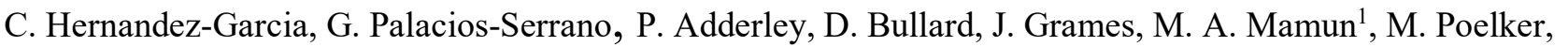

\begin{abstract}
A direct current (dc) high voltage photo-emission electron gun operating at $130 \mathrm{kV}$ is utilized at the Jefferson Lab (JLab) Continuous Electron Beam Accelerator Facility to generate spin-polarized electrons for nuclear physics experiments. Over the past decade, JLab has tested and implemented inverted-geometry ceramic insulators in photoguns, connecting the cathode electrode in vacuum to the high voltage power supply using commercial high voltage cables. The results of those tests showed that breakdown voltage was increased using triple-point shielding electrodes and bulk-doped insulators that allow charge drainage. This contribution describes ongoing work to develop a robust insulatorcable connector for reliably applying $500 \mathrm{kV}$ de to a future polarized beam photogun operating at $350 \mathrm{kV}$ without field emission.
\end{abstract}

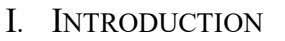

ए

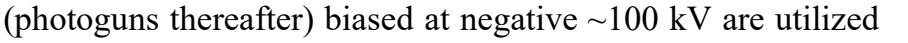
ए

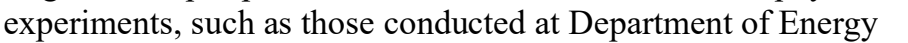

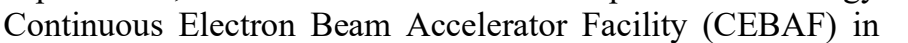

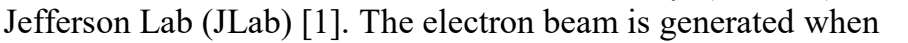

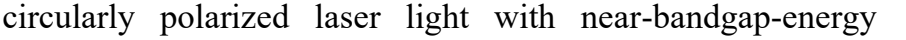

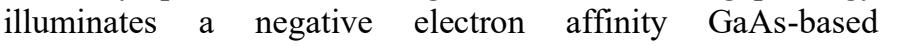

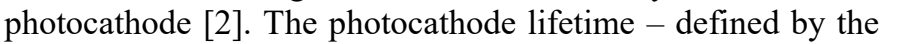

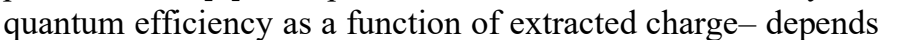

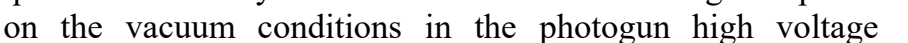

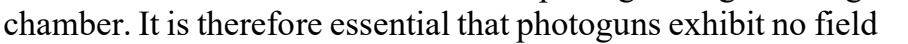

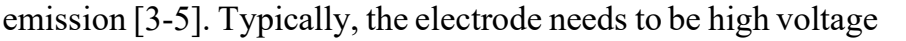

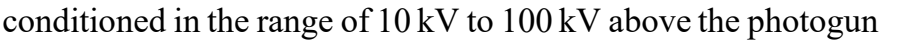
ए

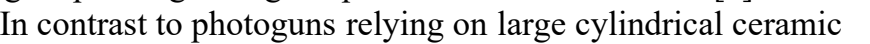

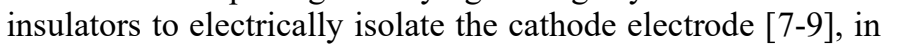

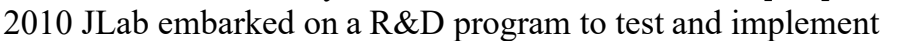

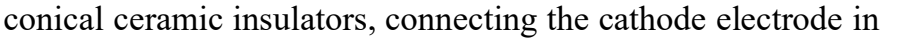

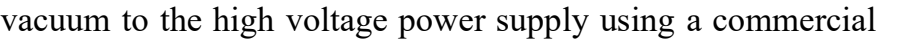

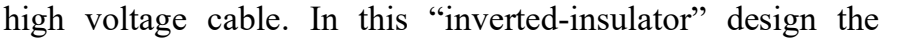

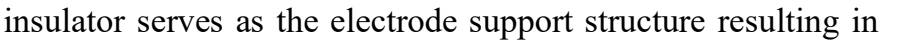

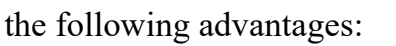

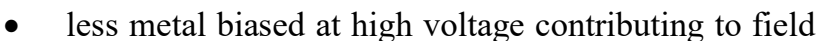

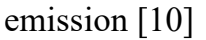

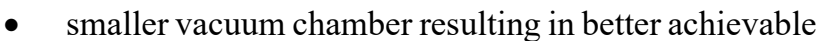
पाएणाए

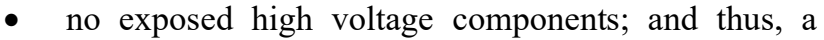

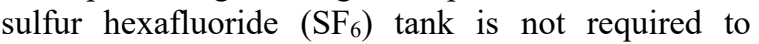

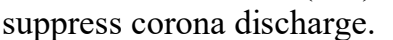

ए ए

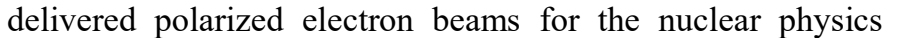

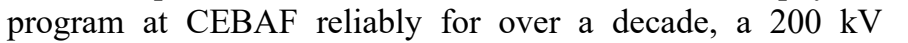

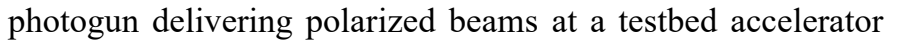

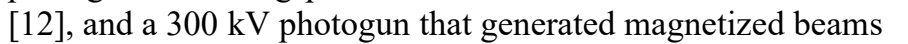

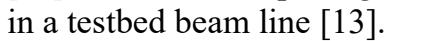

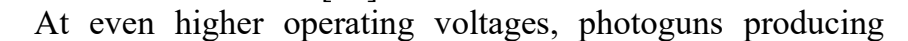

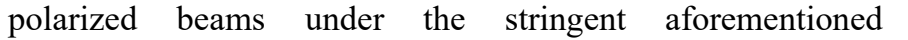

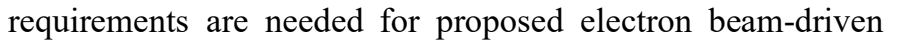

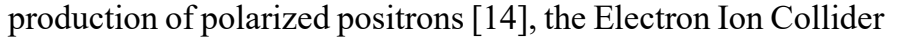

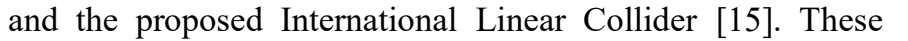

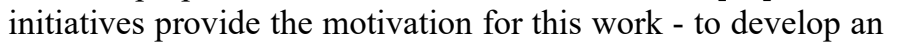

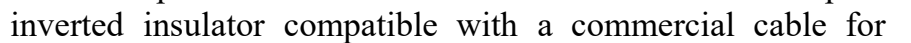

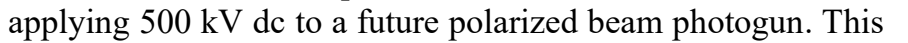

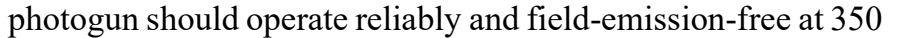

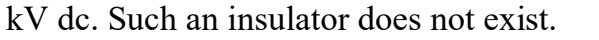

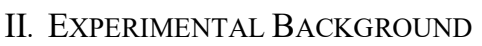

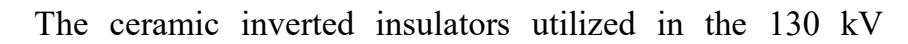

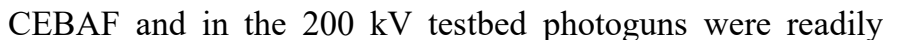

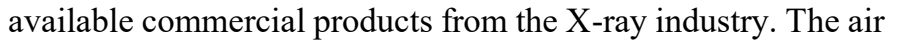

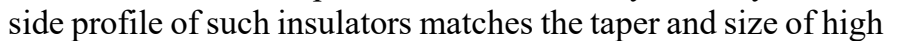

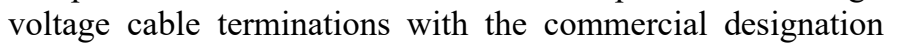

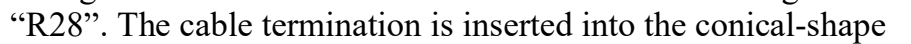

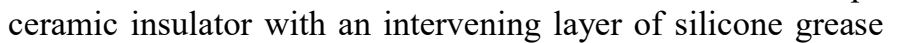

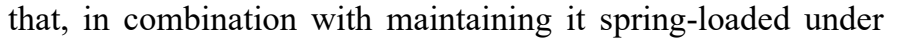

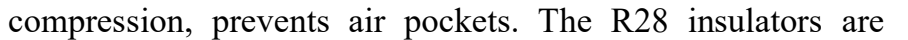

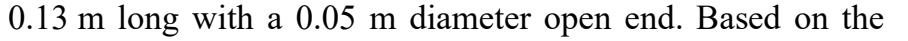

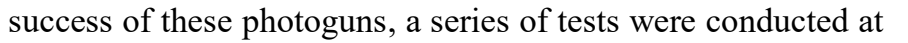

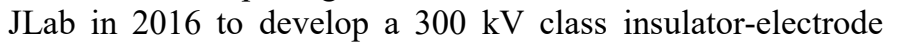




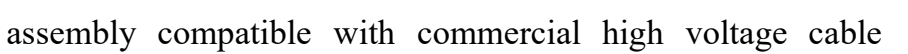

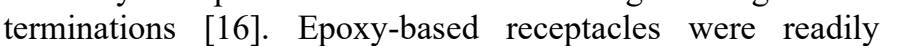

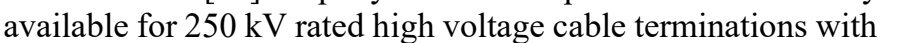

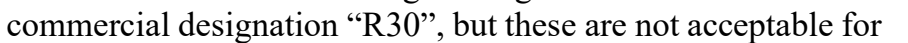

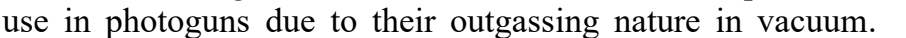

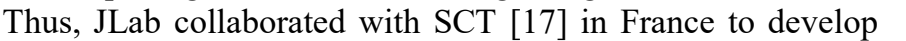

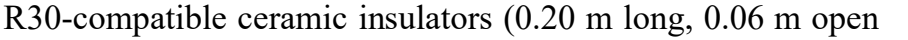

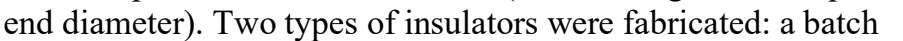

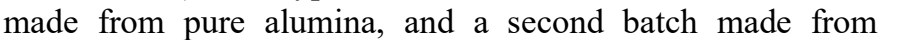

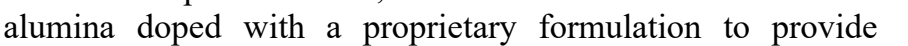

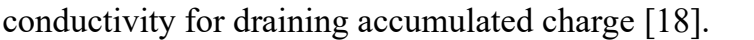

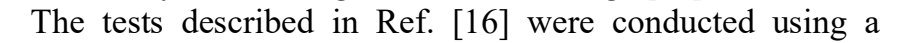

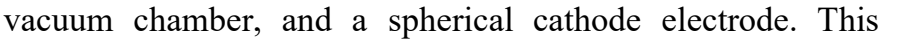

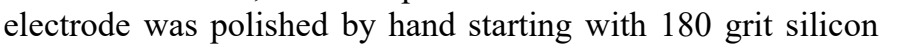

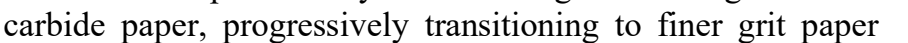

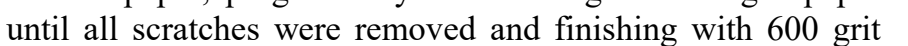

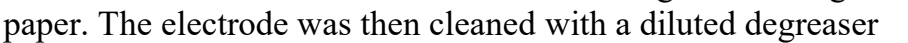

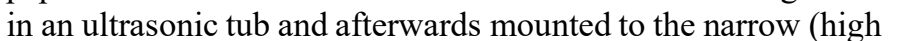

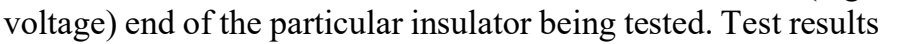

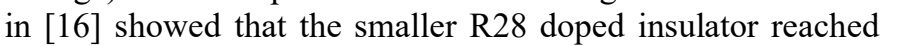

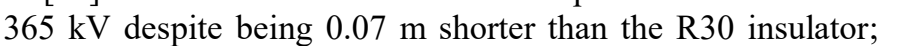

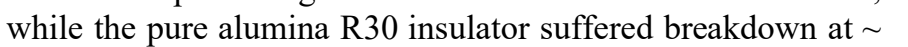

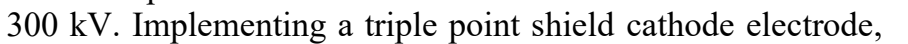
पाm

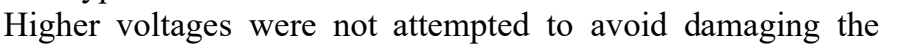
एवाणाणाए

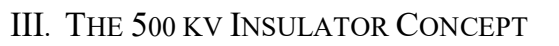

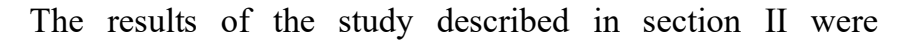

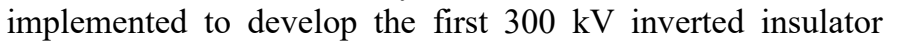

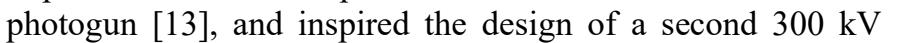

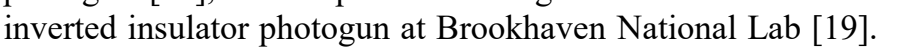

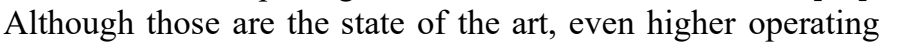

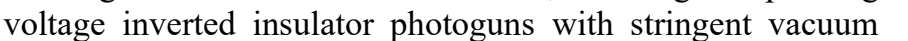

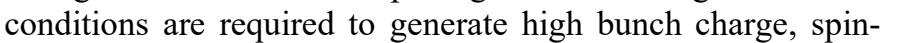

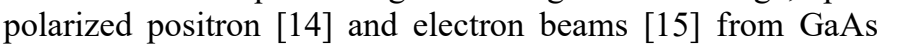

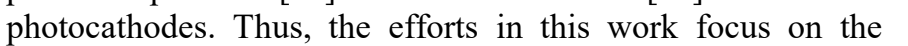

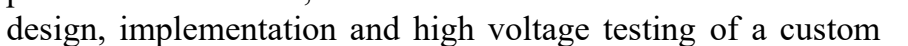

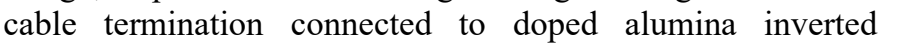

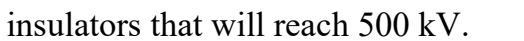

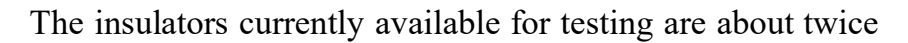

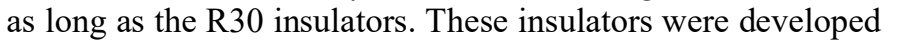

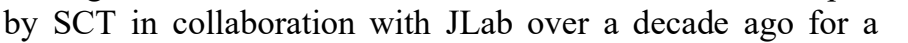

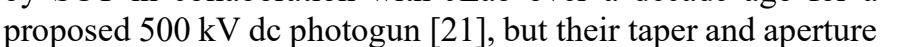

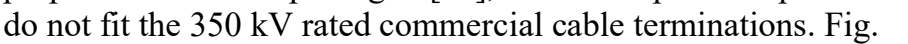

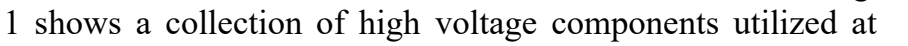

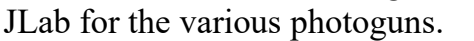

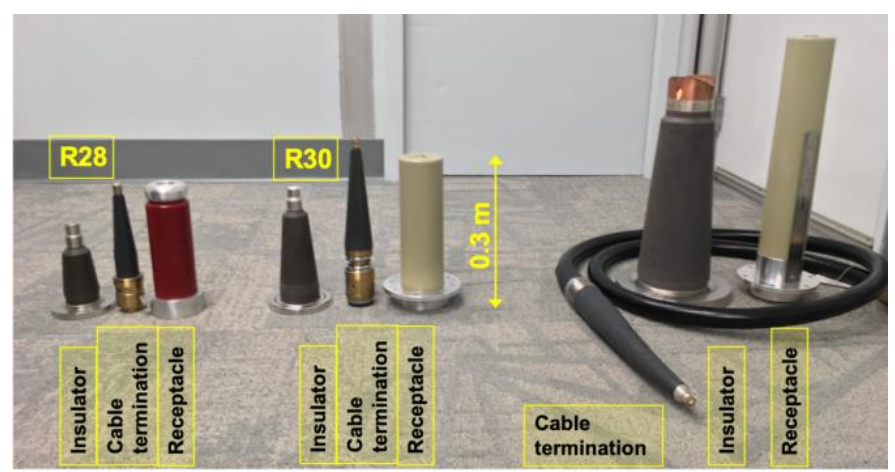

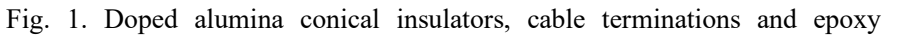

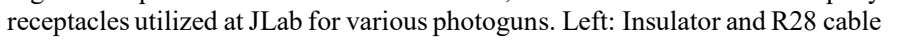

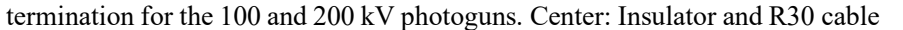

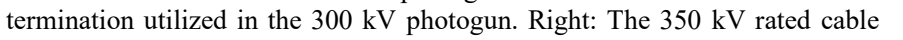

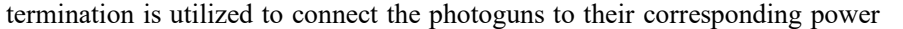

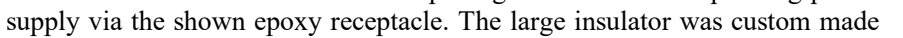
ए एव

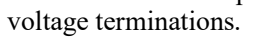

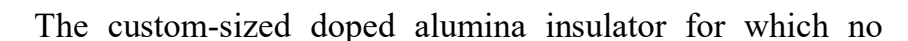

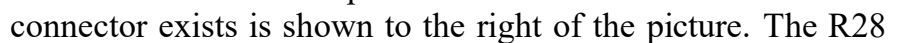

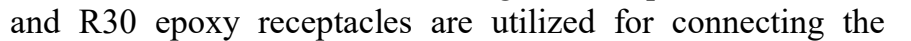

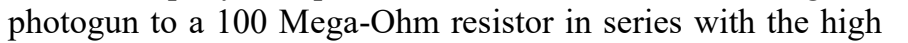

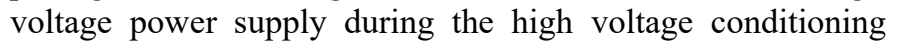

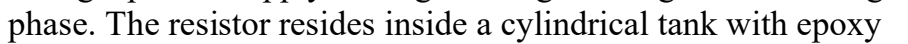

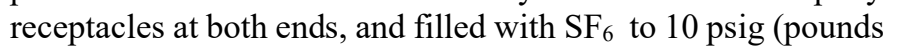

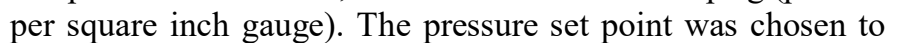

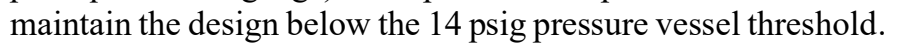

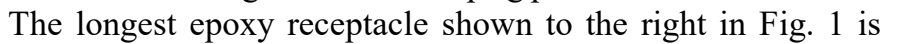

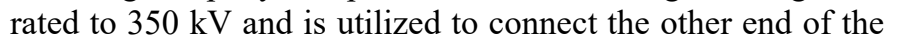

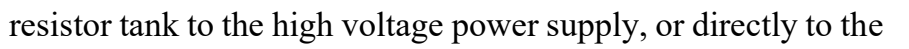

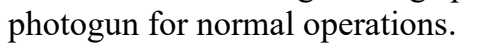

\section{A. Modified $350 \mathrm{kV}$ rated epoxy receptacle with $\mathrm{SF}_{6}$ intervening layer}

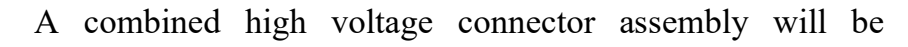

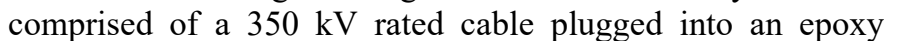
एण

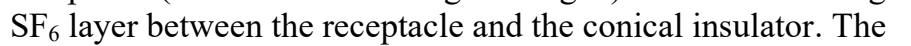

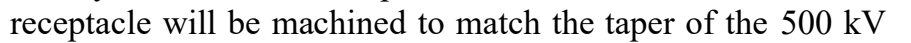
एण

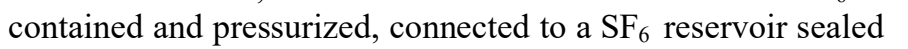

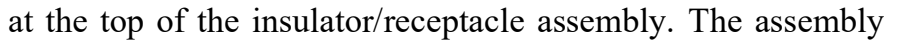

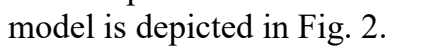




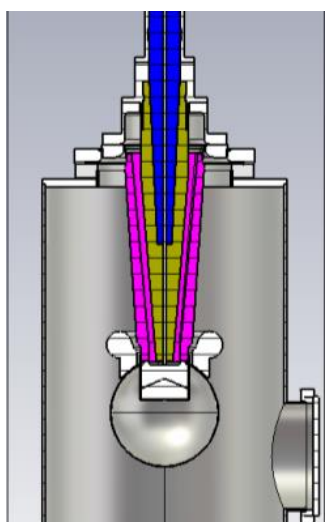

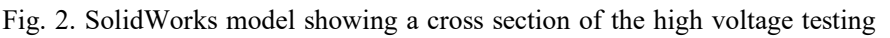

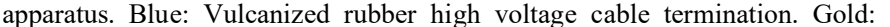

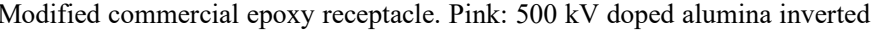

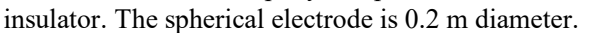

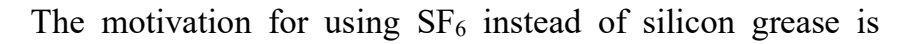

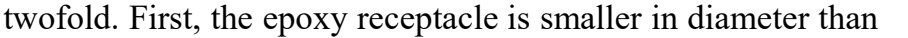

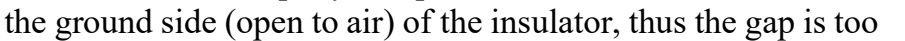

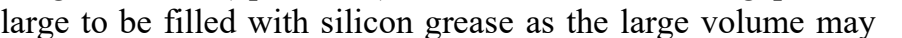

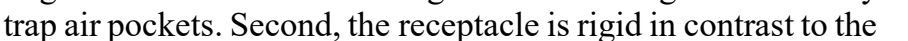

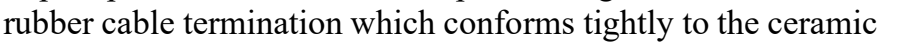

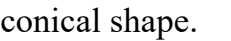

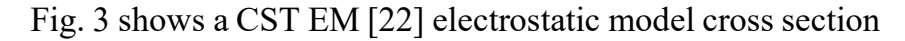

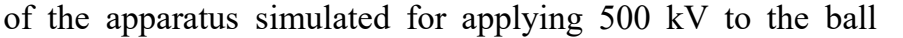
पाणाणा
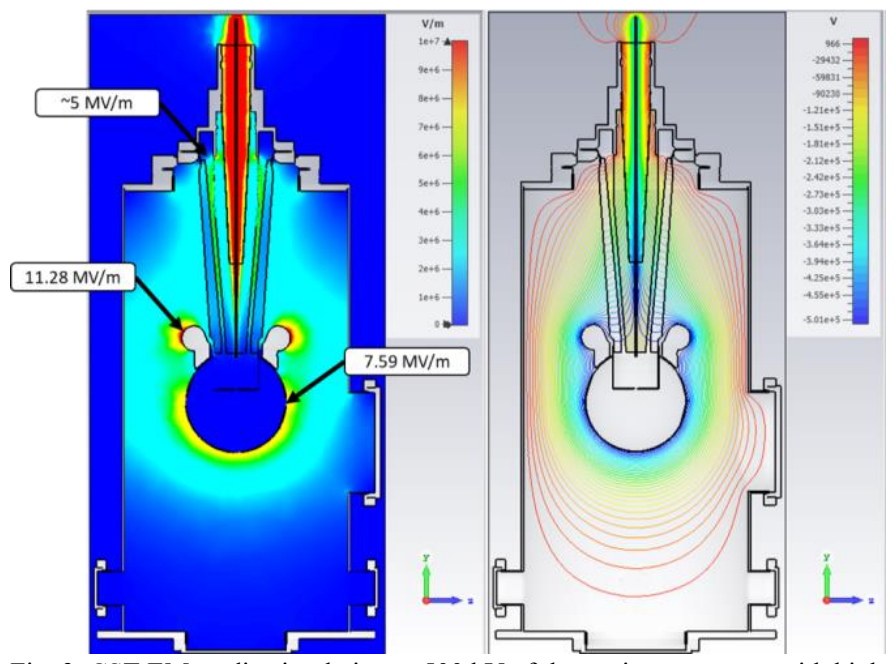

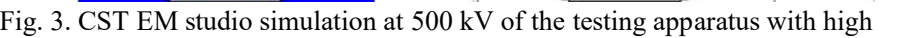

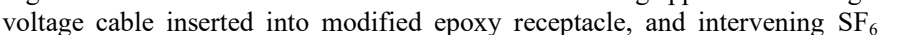

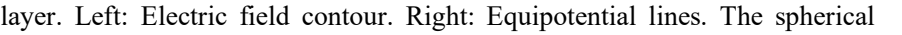

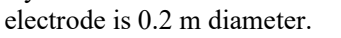

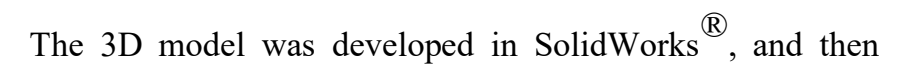

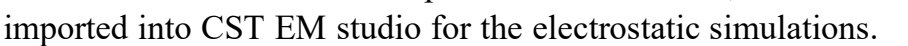

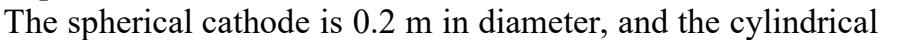

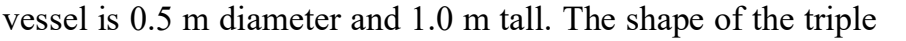

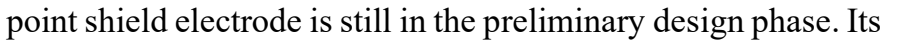
पण

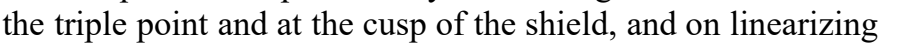
एण

\section{B. Custom high voltage cable termination}

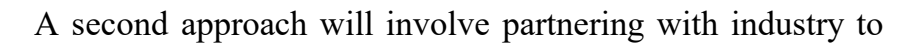

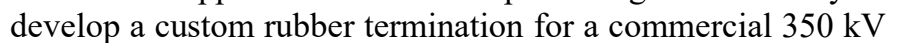

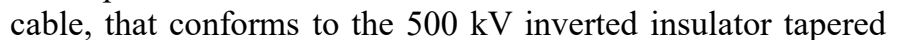

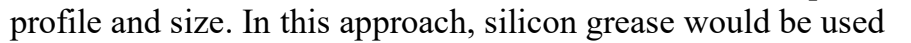

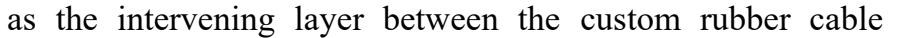
ए

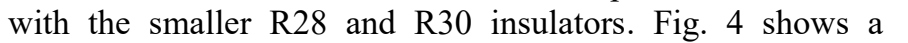

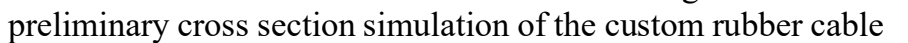

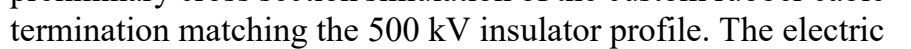

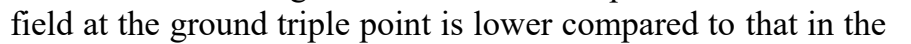

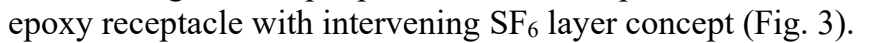

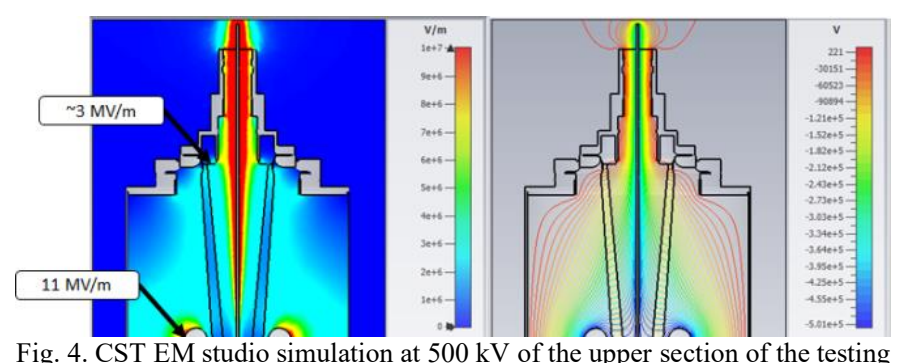

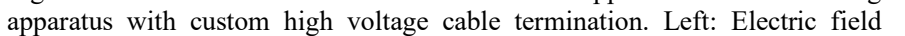

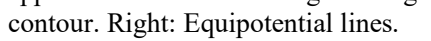

\section{Custom $500 \mathrm{kV}$ insulator design compatible with commercial cable termination}

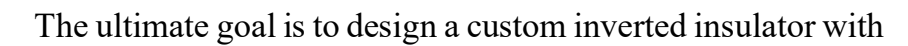

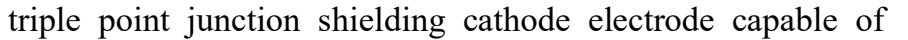

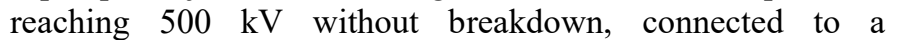

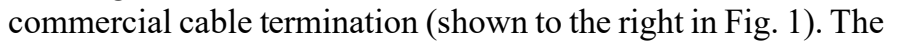

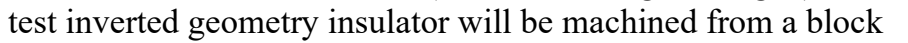

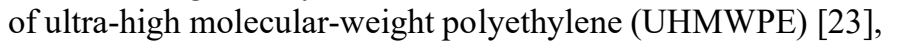

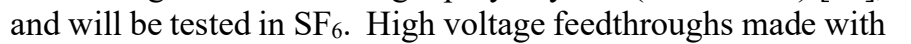

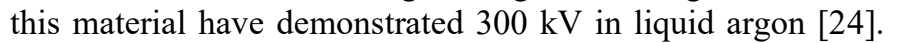

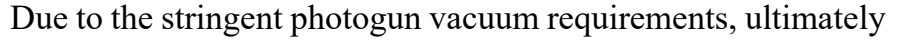

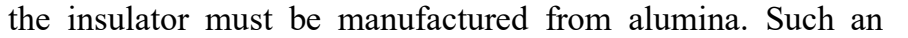

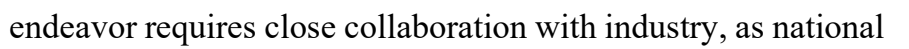

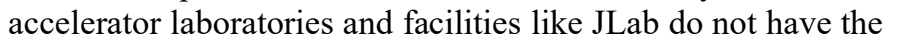
ए ए1

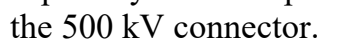

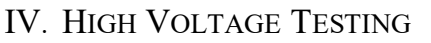

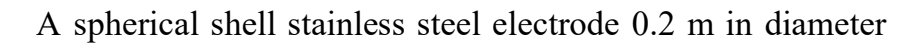

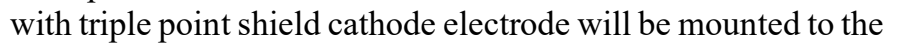

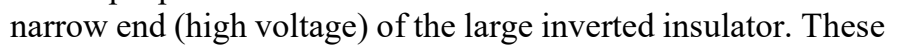

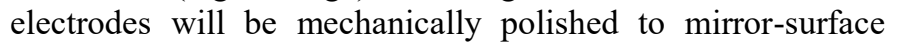
ए

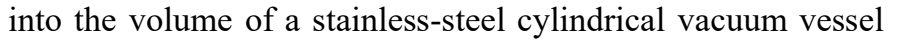

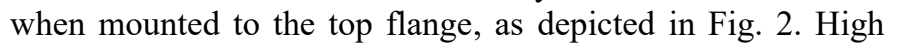

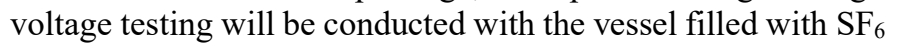

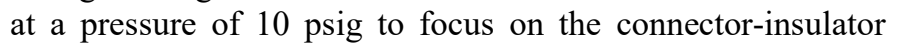

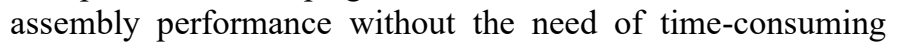

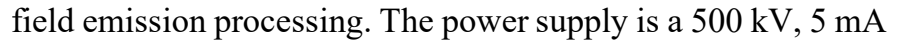




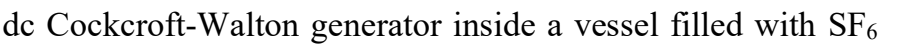

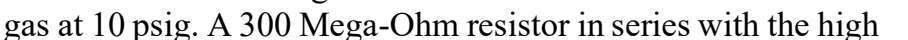

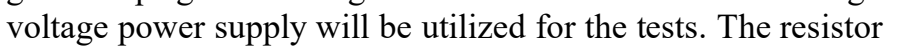

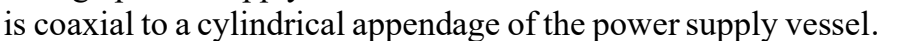

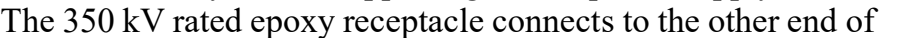

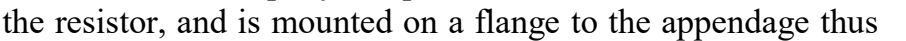

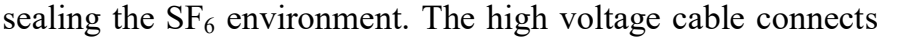

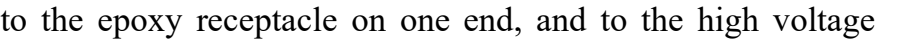

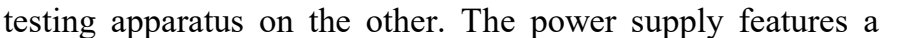

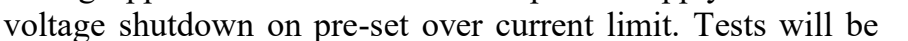

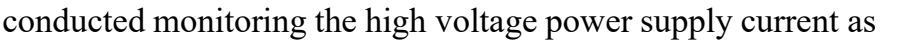

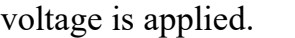

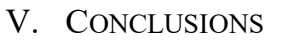

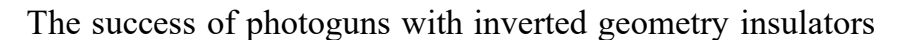

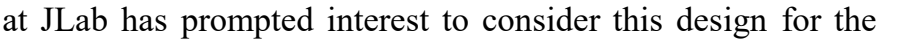

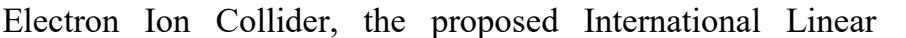

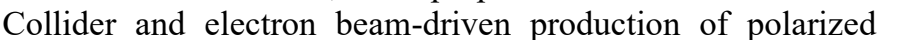

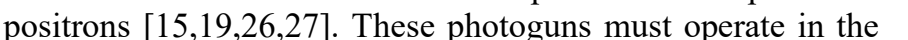

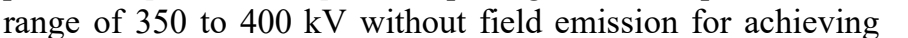

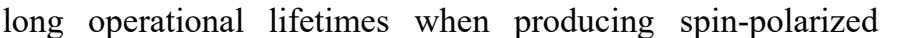

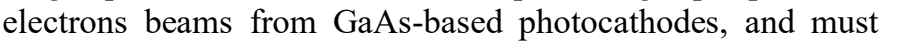

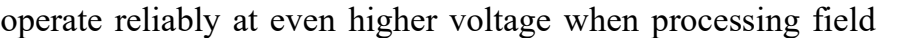

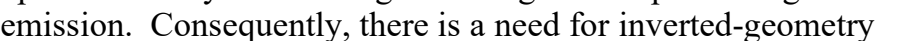

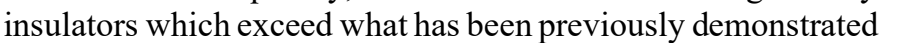

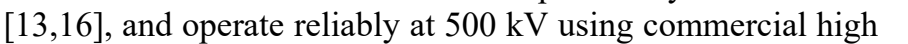

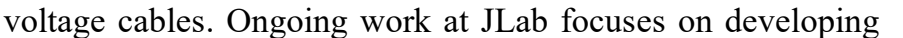

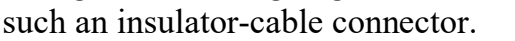

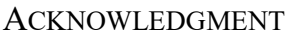

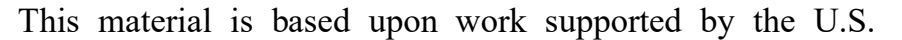

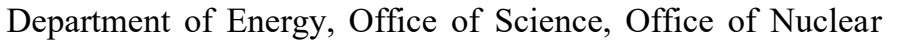

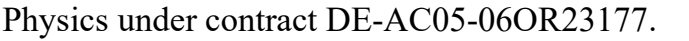

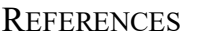

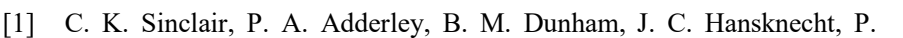

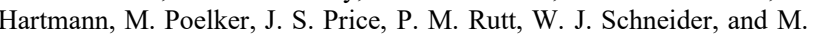

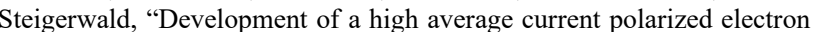

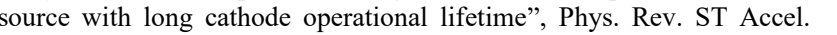

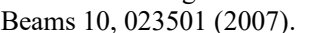

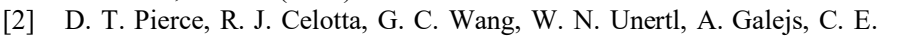

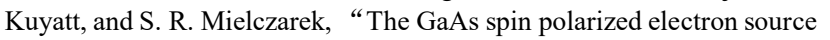

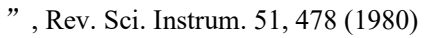

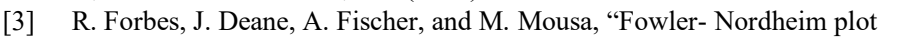

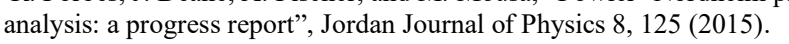

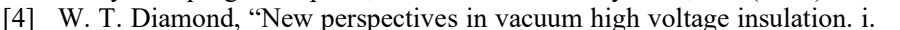

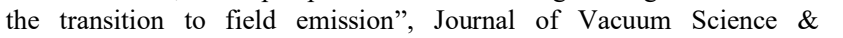

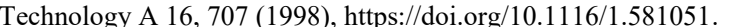

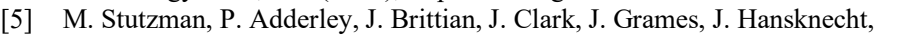

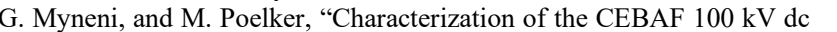

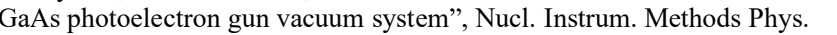

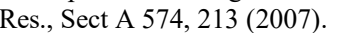

ए

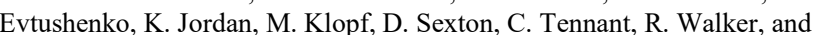

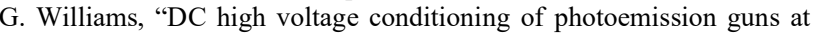

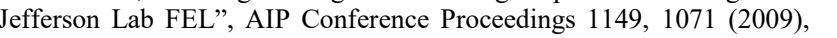

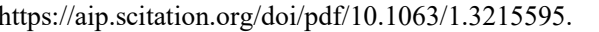

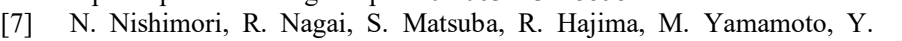

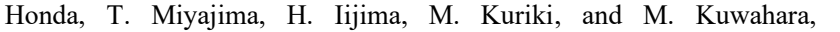

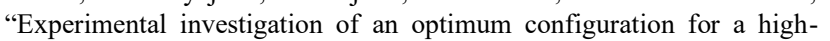

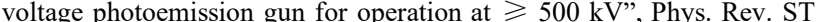

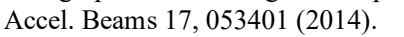

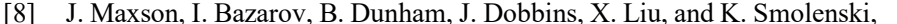

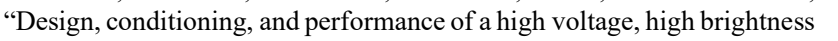

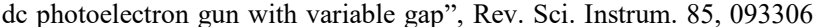

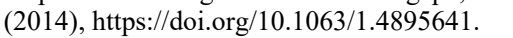

त

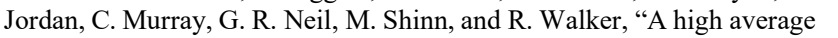

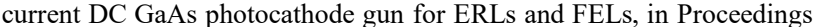

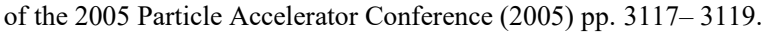

प

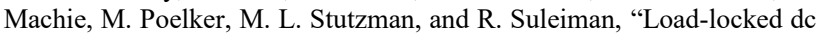

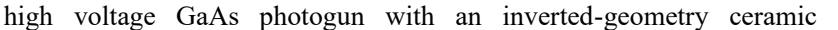

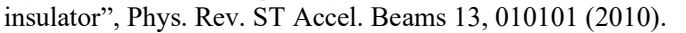

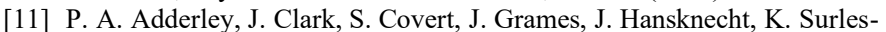

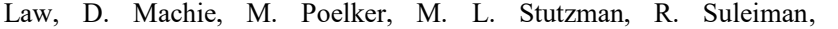
“

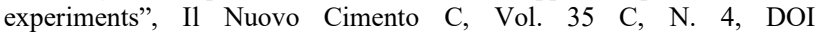

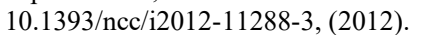

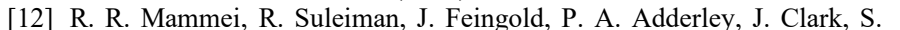

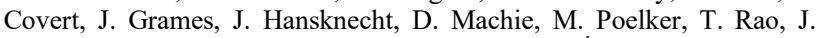

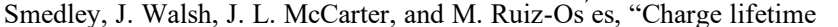

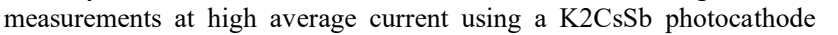

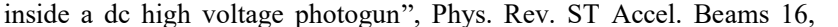

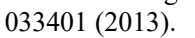

(1)

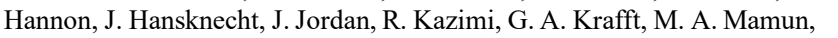

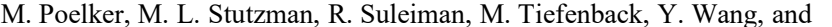

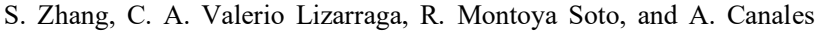

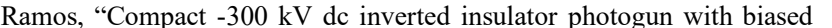

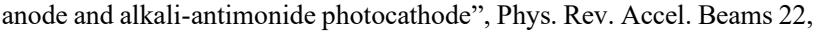

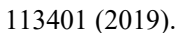

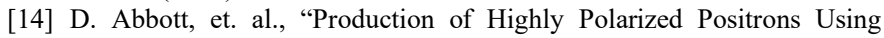
Polarized Electrons at MeV Energies", Phys. Rev. Lett., 116, 214801 पाIIIIII)

$\square \square \mathrm{V}$. Shiltsev, and F. Zimmermann, "Modern and future colliders", $\square \square \square$

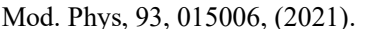

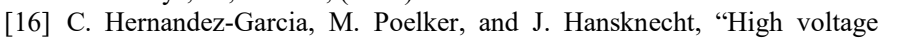

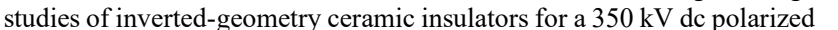

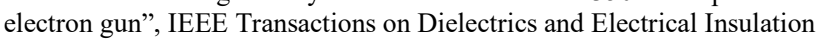

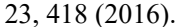

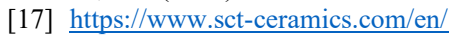

YY. J. Lei, B. H. Tang, X. J. Huang, Y. Huang, X. F. You, M. Zeng, “Effects

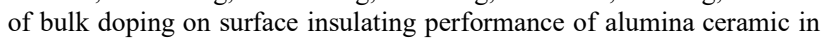
vacuum”, IEEE Trans. Dielectr $\square 11 \mathrm{II}$

[19] E. Wang, "Polarized Electron Source R\&D for the EIC", International

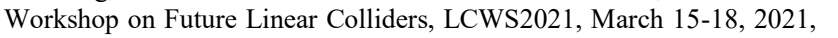

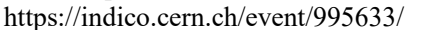

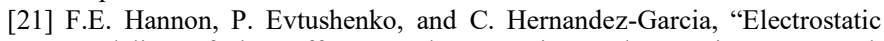
$\square \square \amalg$ ng of the Jefferson Laboratory inverted ceramic gun", Int'l.

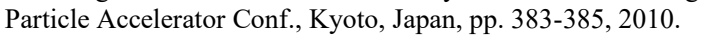

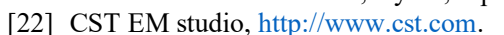

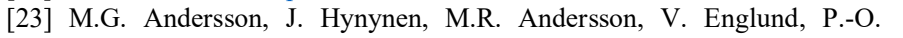

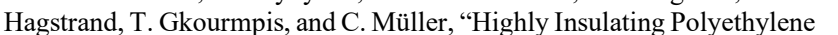

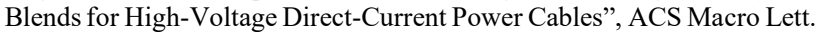

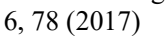

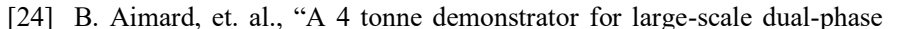

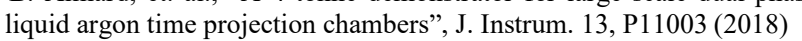

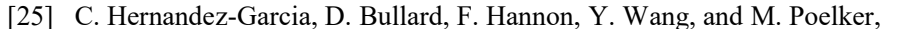
“

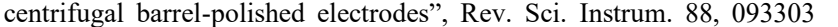
पा1十10

미 J. Dumas, J. Grames, and E. Voutier, "A Polarized Positron Source for CEBAF", AIP Conference Proceedings 1160

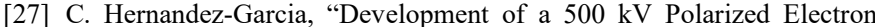

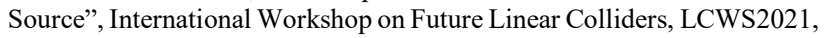

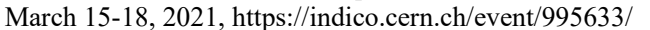

